\title{
Nature Clinical Practice Urology: one year on
}

\author{
Alexandra M Hay and Peter T Scardino
}

Nature Clinical Practice Urology has now reached its 13th issue. After one year, our aims remain the same: to deliver timely, interesting and informative, peer-reviewed articles highlighting topics of interest to practicing urologists, distilling the most relevant research and presenting it in an easily digestible format in order to save our readers time. Since the launch of our first issue in November 2004, we have been delighted with the response from our authors and peer-reviewers; together with our expert international Advisory Board they have helped us to achieve the standard of high-quality, timely articles that we have been striving for. In the last year we have published around 150 peer-reviewed articles written by leading experts and have received over 200 invited articles.

One year into publication we have only scratched the surface of the issues that are of interest to urologists today, and we are continuing to develop our content to ensure coverage of all urologic subspecialties and to include new types of articles. This issue of the journal includes our first Primer Review. These educational reviews aim to provide straightforward jargon-free explanations of areas of special interest to urologists, covering subjects such as statistical methods, new diagnostic techniques, and good clinical practice. In this issue Chris Chapple reviews the use of questionnaires and urodynamic investigations in the diagnosis of lower urinary tract dysfunction. Other Primer Reviews planned for the future include series on the use of statistics in clinical practice and on modern imaging techniques.

A new review type that we plan to introduce is Surgery Insights. These articles will focus on particular surgical procedures or techniques and discuss the advantages and limitations of different approaches. Authors will describe modifications to techniques that may improve perioperative and postoperative outcomes.

Through Practice Points we offer a unique way of delivering expert opinions on recently

....our aims
remain the
same: to
deliver timely,
interesting and
informative,
peer-reviewed
articles
highlighting
topics of
interest to
practicing
urologists...

published research of significance that may shape current practice. These popular articles provide a time-saving resource by highlighting developments in the field accompanied by authoritative commentaries on significant papers of particular interest. One of the most successful, and well appreciated features of Nature Clinical Practice Urology is the way that we approach Case Studies. Rather than presenting anecdotal reports of rare presentations of particular diseases, we aim to publish case studies describing how to best manage patients with common problems that urologists encounter on a regular basis. The aim is to provide insight into diagnostic and therapeutic management of common conditions and diseases, providing a solid basis for continuing medical education (CME). CME credits for Nature Clinical Practice journals can be obtained by submitting answers to the CME questions on the Vanderbilt University CME website (http://www.mc.vanderbilt.edu/medschool/ $\mathrm{cme} /$ ). We welcome Case Study proposals for consideration, which can be submitted online (http://mts-ncpu.nature.com) or by email (ncpuro@nature.com).

The Nature Clinical Practice division of Nature Publishing Group has proven to be a successful new venture, and we are delighted to announce that Nature Clinical Practice Urology has been accepted for inclusion in Medline, and our full archive should be available for searching via Medline soon. Following the success of the first four Nature Clinical Practice journals in cardiovascular medicine, gastroentorology and hepatology, oncology, and urology, four more speciality journals are being launched this month, demonstrating the continued commitment that Nature Publishing Group has to providing high-quality clinical journals of relevance to practicing physicians.

Thank you for your continued interest in the journal. We look forward to delivering more high-quality content to you in the future. 